\title{
HOMOGENEOUS POSITIVELY PINCHED RIEMANNIAN MANIFOLDS
}

\author{
BY NOLAN R. WALLACH
}

Communicated by M. H. Protter, January 26, 1970

1. Introduction. The purpose of this paper is to announce several results on homogeneous positively pinched manifolds. We give, modulo two examples, a complete classification of even dimensional homogeneous spaces that admit positively pinched homogeneous Riemannian structures (see $\$ 5$, Theorem 5.2). These spaces are all diffeomorphic with rank one symmetric space except for two possible exceptions $\mathrm{SU}(3) / T$ ( $T$ the maximal torus in $\mathrm{SU}(3))$ and

$$
\mathrm{Sp}(3) / \mathrm{SU}(2) \times \mathrm{SU}(2) \times \mathrm{SU}(2) .
$$

2. Preliminary results. Let $G$ be a compact connected Lie group and let $K$ be a closed subgroup of $G$. Let $M=G / K$ and suppose that $M$ carries a $G$-invariant positively pinched Riemannian structure (that is all sectional curvatures are bounded below by a positive constant).

Proposition 2.1. If $M$ is even dimensional then $G$ is semisimple. If $M$ is odd dimensional then $G$ is either semisimple or $G$ has a onedimensional center.

This result is proved by analyzing directly the curvature of $M$ as a 4 -linear form on a suitably chosen $\operatorname{Ad}(K)$-invariant complement $\mathfrak{p}$ to the Lie algebra of $K$, in the Lie algebra of $G, \mathfrak{g}$ (in particular $\mathfrak{p}$ is chosen so that it contains the center of $\mathfrak{g}$ ). The even dimensional statement will be considerably strengthened in the next section.

The next result indicates the difficulties in using a Lie theoretic, algebraic approach to the general study of homogeneous positively pinched Riemannian manifolds.

Proposition 2.2. Let $(G, K)$ be as in Proposition 1. Let $T$ be a maximal torus in $K$. Let $C(T)$ be the centralizer of $T$ in $G$. Let $p_{0}=e K$ (the identity coset in $M$ ) and let $M_{T}=C(T) p_{0}$. Then

(1) $M_{T}$ is totally geodesic in $M$.

(2) $C(T) / T$ is either one dimensional or semisimple.

AMS Subject Classifications. Primary 5372, 5366.

Key Words and Phrases. Homogeneous, Riemannian structure, Killing field, Lie group, isometry. 
We propose

CoNJECTURE. Let $G$ be a compact, connected, simply connected Lie group. Suppose that $G$ has a left invariant positively pinched Riemannian structure. Then $G=\mathrm{SU}(2)$.

If the conjecture were true then Proposition 2.1 and Proposition 2.2 would imply

Corollary to ConJecture. Let $G$ be a compact connected Lie group and let $K$ be a closed subgroup of $G$ such that $G$ acts faithfully on $M$ $=G / K$ and such that $M$ admits a G-invariant positively pinched Riemannian structure.

(i) If $M$ is even dimensional then the rank of $K$ is equal to the rank of G.

(ii) If $M$ is odd dimensional then the rank of $G=$ rank of $K+1$.

The above corollary to the conjecture is in fact equivalent to it. We will see in $\$ 3$ that assertion (i) of the corollary is true and a direct consequence of a result of Berger [2] (see also Weinstein [6]).

\section{Implications of a result of Berger.}

Proposition 3.1. Let $M$ be an even dimensional, connected, orientable, positively pinched Riemannian manifold. Let $G$ be a compact connected Lie group acting by isometries effectively and transitively on $M$. Let $K$ be the isotropy group, in $G$, of a point in $M$. Then $G$ is a simple center free Lie group and $G$ and $K$ have the same rank.

This result follows directly from a result of Berger [2] which says that every Killing field on an even dimensional positively pinched manifold must have a zero. See also Weinstein [2] for a strengthening of this result.

Using Proposition 3.1 and a result of O'Neil [4], we have

Corollary 3.1. Let $G$ be a compact simply connected lie group admitting a positively pinched left invariant Riemannian structure then $G$ is odd dimensional and simple.

4. Main lemma. Let $G, K$ be as in the beginning of $\$ 2$. Let $M=G / K$ and assume that $M$ is even dimensional. Then, by Proposition 3.1, $G$ has a maximal torus, $T$, contained in $K$. Let $\Delta$ be the root system (written additively) of $G$ relative to $T$ and let $\Delta_{1}$ be the root system of $K$ relative to $T$. Then $\Delta_{1} \subset \Delta$. Let $\Delta-\Delta_{1}$ denote the complement to $\Delta_{1}$ in $\Delta$.

Lemma 4.1. If $\alpha, \beta \in \Delta-\Delta_{1}$ and $\alpha \neq \pm \beta$ then $\alpha+\beta$ or $\alpha-\beta \in \Delta$. 
5. The classification theorem. Let $G$ be a compact, connected, simply connected, simple, Lie group and let $K$ be a closed subgroup of $G$ such that $G$ and $K$ share a maximal torus $T$. Let $\Delta$ be the root system of $G$ relative to $T$ and let $\Delta_{1}$ be the root system of $K$ relative to $T$. Then $(G, K)$ is said to satisfy condition (A) if

(A) $\alpha, \beta \in \Delta-\Delta_{1}, \alpha \neq \pm \beta$ implies $\alpha+\beta$ or $\alpha-\beta \in \Delta$.

THEOREM 5.1. The only pairs $(G, K)$ as above satisfying condition (A) are

1. $(\mathrm{SU}(n+1), \mathrm{U}(n))$ and $G / K=C P^{n}$ complex $n$ dimensional projective space.

2. (SU(3), $T)$ where $T$ is a maximal torus of $\mathrm{SU}(3)$.

3. (Spin $(2 n+1), \operatorname{Spin}(2 n))$ and $G / K=S^{n}$ the $n$ sphere.

4. $(\mathrm{Sp}(n), \mathrm{Sp}(n-1) \times \mathrm{SU}(2))$ and $G / K$ is $n$ dimensional quaternionic projective space.

5. $\left(\mathrm{Sp}(n), \operatorname{Sp}(n-1) \times T^{\prime}\right)$ where $T^{\prime}$ is a circle group and $G / K$ is diffeomorphic with $\mathrm{C} P^{2 n-1}$.

6. $(\mathrm{Sp}(3), \mathrm{SU}(2) \times \mathrm{SU}(2) \times \mathrm{SU}(2))$.

7. $\left(F_{4}, \operatorname{Spin}(9)\right)$ and $G / K$ is the Cayley plane.

8. $\left(G_{2}, \mathrm{SU}(3)\right)$ and $G / K$ is diffeomorphic with $S^{6}$.

The proof of this result is given by a case by case check of the compact simply connected simple Lie groups, using the results of [4] and the observation that if $(G, K)$ satisfies condition (A) and if $G \supset K_{1} \supset K, K_{1}$ a closed subgroup of $G$ then $\left(G, K_{1}\right)$ satisfies condition (A).

Combining Proposition 3.1, Lemma 4.1 and Theorem 5.1, we have

THeOREM 5.2. Let $M$ be a connected, simply connected, even dimensional, positively pinched Riemannian manifold. Let $G$ be a connected, closed transitive subgroup of the isometry group of $M$. Let $\tilde{G}$ be the universal covering group of $G$ and let $\tilde{K}$ be the isotropy group of a point in $M$ relative to the induced action of $\tilde{G}$ on $M$. Then $(\tilde{G}, \tilde{K})$ is one of the pairs 1-8 of Theorem 5.1.

Note. Except for 2, 6 the pairs above in Theorem 5.1 are exactly the pairs that Berger [1] found in the even dimensional naturally reductive (that is, the Riemannian structure comes from a biinvariant metric on $G$ ) case.

Corollary 5.1. Let $M$ be as in Theorem 5.2. Then $M$ is either diffeomorphic to a rank 1 symmetric space or $M$ may be one of $\mathrm{SU}(3) / T$, $\mathrm{Sp}(3) / \mathrm{SU}(2) \times \mathrm{SU}(2) \times \mathrm{SU}(2)$.

We are unable at this point to prove (or disprove) that the above 
exceptional examples have homogeneous positively pinched metrics. Using Proposition 2.1 and Theorem 5.2 we have

Corollary 5.2. Let $G, K$ be as in Proposition 2.1. If $G$ is not semisimple then $G=\mathrm{U}(n)$ or $G=T^{\prime} \times \mathrm{Sp}(n)$.

\section{REFERENCES}

1. M. Berger, Les variêtes riemanniennes homogènes normales simplement connexes $a$ courbure strictemente positive, Ann. Scuola Norm. Sup. Pisa (3) 15 (1961), 179-246. MR 24 \#A2919.

2. - Trois remarques sur les variêtés riemannienes à courbure positive, $\mathrm{C} . \mathrm{R}$. Acad. Sci. Paris Sér. A-B 263 (1966), A76-A78. MR 33 \#7966.

3. S. Kobayashi and K. Nomizu, Foundations of differential geometry. Vol. II, Interscience, New York, 1969.

4. B. O'Neill, The fundamental equations of a submersion, Michigan Math. J. 13 (1966), 459-469. MR 34 \#751.

5. N. Wallach, On maximal subsystems of root systems, Canad. J. Math. 20 (1968), 555-574. MR 38 \#1135.

6. A. Weinstein, $A$ fixed point theorem for positively curved manifolds, J. Math. Mech. 18 (1968/69), 149-153. MR 37 \#3478.

Rutgers University, New Brunswick New Jersey 08903 